\title{
Random Waypoint Considered Harmful
}

\author{
Jungkeun Yoon, Mingyan Liu, Brian Noble \\ Electrical Engineering and Computer Science Department \\ University of Michigan \\ Ann Arbor, Michigan 48109-2122 \\ \{jkyoon, mingyan, bnoble\}@eecs.umich.edu
}

\begin{abstract}
This study examines the random waypoint model widely used in the simulation studies of mobile ad hoc networks. Our findings show that this model fails to provide a steady state in that the average nodal speed consistently decreases over time, and therefore should not be directly used for simulation. We show how unreliable results can be obtained by using this model. In particular, certain ad hoc routing metrics can drop by as much as $40 \%$ over the course of a 900-second simulation using the random waypoint model. We give both an intuitive and a formal explanation for this phenomenon. We also propose a simple fix of the problem and discuss a few alternatives. Our modified random waypoint model is able to reach a steady state and simulation results are presented.
\end{abstract} ing.

Index Terms - random waypoint model, mobility, ad hoc rout-

\section{INTRODUCTION}

Mobile systems are characterized by the movement of their constituents. The nature of movement - its speed, direction, and rate of change - can have a dramatic effect on protocols and systems designed to support mobility. Unfortunately, movement in the physical world is often unrepeatable. Live use of a mobile system can provide meaningful insight, but cannot form the sole basis of experimental evaluation.

Instead, the mobile computing community has turned to simulating the movement of nodes and users. Of course, one must derive a model of movement to drive such a simulation. By far the most common of these is the random waypoint model. This model was first used by Johnson and Maltz in the evaluation of Dynamic Source Routing (DSR) [1], and was later refined by the same research group [2]. The refined version has become the de facto standard in mobile computing research. For example, ten papers in ACM MobiHoc 2002 considered node mobility, and nine of them used the random waypoint model.

In this model, nodes in a large "room" choose some destination, and move there at a random speed uniformly chosen from $\left(0, V_{\max }\right]$, where $V_{\max }$ is the maximum speed of the simulation. Often, the model is described as having an average speed of $\frac{V_{\max }}{2}$. This model is expected to maintain this average speed as the simulation progresses, and simulation results are almost always in the form of an average over a period of time. Such averages only make sense if the simulation reaches a steady state. Unfortunately, this is not the case. The fact is that as simulated time progresses, the collection of nodes moves more slowly; more and more nodes become "stuck" travelling long distances at low speeds. Thus the model fails to provide a steady state in terms of average speed. The overheads and performance of mobile systems usually depend strongly on node mobility. In light of this, random waypoint can generate misleading or incorrect results. In particular, time-average results change drastically over time; the longer we run the simulation, the further results deviate.

This paper presents an analysis of a generalized random waypoint model that predicts the average speed of nodes in the simulation. This analysis closely matches the actual model. There are many ways to correct the original model. One simple solution is to limit the minimum speed, as well as the maximum. This paper compares this simple improvement to the original model, and demonstrates its marked improvement in stability over the course of the simulation. We also explore the impact of instability on two ad hoc routing protocols, DSR [1], [2] and AODV [3], [4]. Either protocol can produce better packet delivery rates and delays, depending on the average speed of the nodes during simulation.

This paper's results highlight our belief that simulation studies must be undertaken with great caution. Many assumptions in simulation models are hidden; they must be carefully examined to ensure that the model behaves as expected. This paper identifies a hidden assumption in the random waypoint mobility model, and proposes a simple technique to produce stable movement patterns that are more suited for simulation studies. Much remains to be done to ensure that mobility models accurately capture our expectations of how mobile users actually move.

The rest of the paper is organized as follows. In Section II we describe in detail the observed problem with the random waypoint model via an intuitive explanation and a formal analysis. Section III provides a simple improvement to the original model and presents simulation results. Discussion on alternative solutions and related works are given in Section IV. Section V concludes the paper.

\section{AN IN-DEPTH LOOK INTO THE RANDOM WAYPOINT MODEL}

\section{A. The problem and an intuitive explanation}

The performance measures of ad hoc routing protocols are directly affected by the underlying mobility model used. One of 
the most important parameters of a mobility model is the node speed, either in the form of a constant value or in the form of a certain distribution [2], [4], [5], [6], [7]. Users should be able to adjust this parameter in order to compare the performance of routing protocols under different levels of nodal speed. In doing so, a mobility model is naturally expected to reach a certain steady state in terms of the level of mobility where the average node speed is stabilized around a constant and does not change over time. In the random waypoint model, this average is often believed to be half of the maximum speed - simply because node speeds are chosen from a uniform distribution $\left(0, V_{\max }\right]$ - or some value between 0 and $V_{\max }$ if we consider positive pause time. Moreover, this average is believed to be reached at the onset of the simulation. Based on such expectations, simulation studies often include comparisons by varying values of $V_{\max }$. For each value of $V_{\max }$, simulation results (e.g., routing overhead, packet delivery ratio, etc.) are often in the form of averages over a period of time (e.g., 900 seconds used by studies in [2], [4]).

But are these expectations really justified? We formally define the instantaneous average nodal speed of mobility scenarios generated by the random waypoint model as follows:

$$
\bar{v}(t)=\frac{\sum_{i=1}^{N} v_{i}(t)}{N}
$$

where $N$ is the total (fixed) number of nodes in the scenario, and $v_{i}(t)$ is the speed of the $i^{t h}$ node at time $t$. Using this definition, an example generated by the random waypoint model with maximum speed of $20 \mathrm{~m} / \mathrm{s}$ and zero pause time is plotted in Fig. 1. This is the average over 30 different scenarios and the average speed is calculated every second.

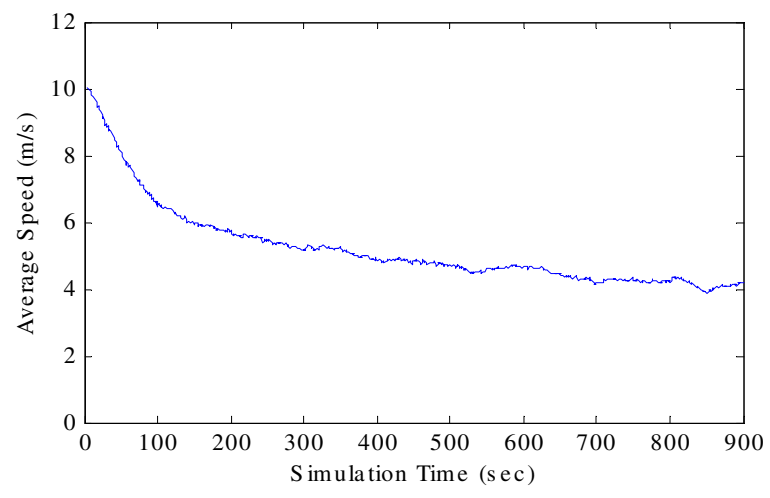

Fig. 1. Average speed decay $(\mathrm{speed}=(0,20] \mathrm{m} / \mathrm{s}$, pause $=0 \mathrm{sec})$

We see that the instantaneous average speed is consistently decreasing. As we will show in the next subsection, this average under the random waypoint model would eventually approach zero! This may happen over a very long period of time, e.g., in the case shown in Fig. 1 the average drops to $4.07 \mathrm{~m} / \mathrm{s}$ at $t$ $=1000 \mathrm{~s}, 3.16 \mathrm{~m} / \mathrm{s}$ at $t=5000 \mathrm{~s}$, and $2.85 \mathrm{~m} / \mathrm{s}$ at $t=10000 \mathrm{~s}$. It decays slowly but consistently. We can easily imagine how erroneous and misleading results can be obtained if we use this model to evaluate the performance of ad hoc routing protocols. We will show specific instances in Section III.

An intuitive explanation for this phenomenon is as follows. The random waypoint model chooses a destination and a speed for a node at random and independently, and the node will keep moving at that speed until it reaches the destination. Given such, a node with a slow speed and a far-away destination may take a long time to finish the trip or may never reach the destination within simulation time. For example, using an area of $1500 \mathrm{~m}$ $\times 500 \mathrm{~m}$ and speed range of $(0,20] \mathrm{m} / \mathrm{s}$, if a destination is chosen $1000 \mathrm{~m}$ away and the speed is chosen to be $0.1 \mathrm{~m} / \mathrm{s}$, then the travel time would be 10000 seconds. If nodes do reach the destination they will be assigned another possibly higher random speed, but nodes like this can be "trapped" to these slow journeys for significant amount of time and therefore dominate the average nodal speed. As the simulation time goes on, on average more and more nodes will be trapped to slower trips, thus causing the speed decay observed in Fig 1. Running the simulation longer causes the average to reduce further. Before we move on to present our fix for this problem, it helps to take a closer look at the probabilistic reasons behind this problem in a more formal way, which we discuss in the next subsection.

\section{B. A formal analysis of the problem}

Here we elaborate the procedure of the random waypoint model. We first choose a rectangular area of size $X_{\max } \times Y_{\max }$, and the total number of nodes $N$ in the area. We then choose a random initial location $(x, y)$ for each node, where $x$ and $y$ are both uniformly distributed over $\left[0, X_{\max }\right]$ and $\left[0, Y_{\max }\right]$, respectively. Every node is then assigned a destination $\left(x^{\prime}, y^{\prime}\right)$, also uniformly distributed over the two-dimensional area, and a speed $v$, which is uniformly distributed over $\left(0, V_{\max }\right]$ (Although often presented as a uniform distribution over $\left[0, V_{\max }\right]$, in actual simulation, e.g., the setdest mobility generation utility in ns-2 [8], zero is always eliminated to avoid division by zero.). $V_{\max }$ is the user-assigned maximum allowed speed. A node will then start travelling toward the destination on a straight line at the chosen speed $v$. Upon reaching the destination $\left(x^{\prime}, y^{\prime}\right)$, the node stays there for some pause time, either constant or randomly chosen from a certain distribution. Upon expiration of the pause time, the next destination and speed are again chosen in the same way and the process repeats until the simulation ends.

For simplicity and clarity of our illustration, we will analyze a modified version of the above model with the following assumptions. We note that our conclusion remains true for the original random waypoint model, but the simplifying assumptions help to isolate and emphasize the key reasons behind the vanishing average nodal speed.

Assumption 1(A1): Instead of confining the nodal movement to a rectangular area of $X_{\max } \times Y_{\max }$, we will assume that nodes move in an unlimited, arbitrarily large area. Given the current location of a node, the destination is chosen uniformly from a circle of radius $R_{\max }$ centered at the current node location. The rationale behind this assumption is that it allows 
us to easily derive the distribution of travel distances. In the original model the travel distance is dependent on the node location due to the limited movement area. We emphasize again that this assumption only helps simplify the analysis but does not change the ultimate conclusion. The boundary effect of the current random waypoint model has been studied in [6], [7], [9]. However, with or without boundary does not fundamentally affect our analysis here.

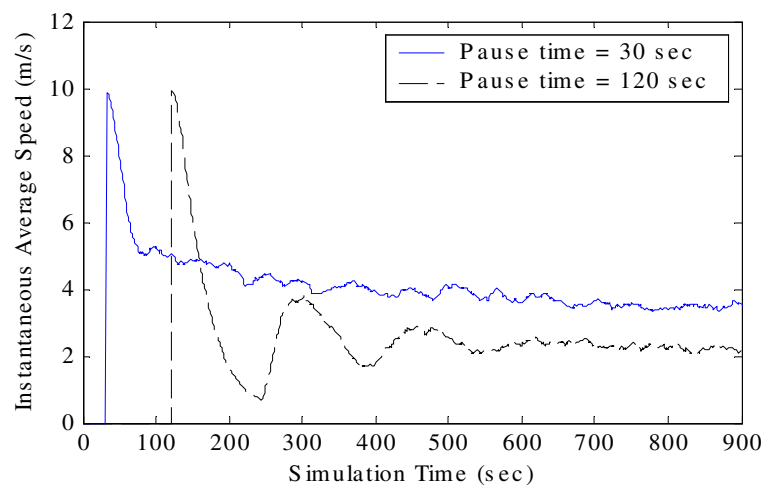

Fig. 2. Average speed decay with various pause time $($ speed $=(0,20])$

Assumption 2(A2): We assume that all pause times are zero. Again this assumption is made because the pause time is not key to the speed decay, and thus eliminating it simplifies the analysis. Fig. 2 shows how the average node speed decays with non-zero pause times. Even though longer pause times lead to fluctuations in the beginning, such effect is gradually reduced and the average node speed (or the envelope of the fluctuation) still decays as time progresses.

Assumption 3(A3): We assume that for each travel the node speed is chosen uniformly from the interval $\left[V_{\min }, V_{\max }\right]$ instead of $\left(0, V_{\max }\right]$, where $V_{\min }>0$. This is because the latter is a limiting case of the former, and thus can be easily derived from results obtained for the former. By this assumption we also imply that the user can specify both the minimum and maximum allowed speeds. Given this assumption the probability density function (pdf) of the nodal speed $V$ is

$$
f_{V}(v)=\frac{1}{V_{\max }-V_{\min }}, \quad V_{\min } \leq v \leq V_{\max } .
$$

Note that the choice of speed and the choice of destination (therefore travel distance) are mutually independent. After some derivation we can obtain the pdfs and expectations for the travel distance and the travel time summarized as follows (see the Appendix for details).

The pdf of the travel distance $R$ is

$$
f_{R}(r)=\frac{2 r}{R_{\max }^{2}}, \quad 0 \leq r \leq R_{\max }
$$

and the expected travel distance is $E[R]=\frac{2}{3} R_{\max }$.

The pdf of the travel time $S$ (note that as a random variable,

$$
\begin{aligned}
& \left.S=\frac{R}{V}\right) \text { is } \\
& f_{S}(s)=\left\{\begin{array}{cl}
\frac{2 s}{3 R_{\max }^{2}}\left(V_{\max }^{2}+V_{\min }^{2}\right. & 0 \leq s \leq \frac{R_{\max }}{V_{\max }} \\
\frac{\left.+V_{\max } V_{\min }\right)}{3\left(V_{\max }-V_{\min }\right)} \frac{1}{s^{2}}- & \frac{R_{\max }}{V_{\max }} \leq s \leq \frac{R_{\max }}{V_{\min }} \\
\frac{2 V_{\min }^{3}}{3 R_{\max }^{2}\left(V_{\max }-V_{\min }\right)} s & s \geq \frac{R_{\max }}{V_{\min }}
\end{array}\right.
\end{aligned}
$$

and the expected travel time is

$$
E[S]=\frac{2 R_{\max }}{3\left(V_{\max }-V_{\min }\right)} \ln \left(\frac{V_{\max }}{V_{\min }}\right) .
$$

This pdf is shown in Fig. 3.

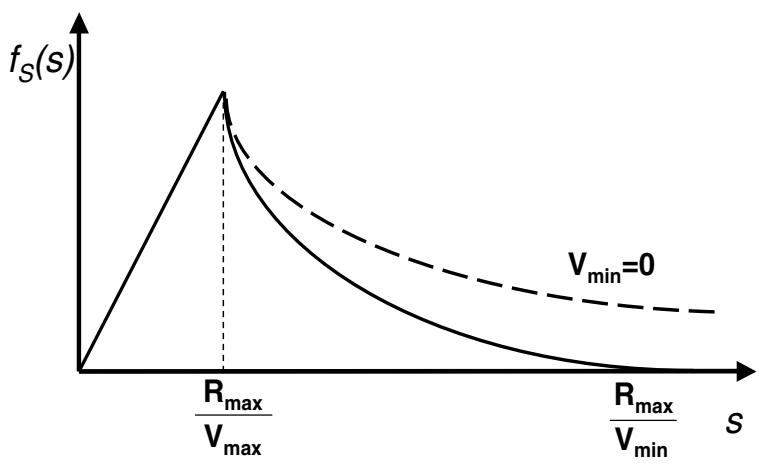

Fig. 3. The pdf of travel time $S$

Given the above results, we can compute the time average of the speed for a given node, $\bar{V}$, as follows, assuming the instantaneous node speed of $v(t)$ at time $t$ (note that since each node moves independently, it suffices to consider a single node):

$$
\begin{aligned}
\bar{V} & =\lim _{T \longrightarrow \infty} \frac{1}{T} \int_{0}^{T} v(t) d t \\
& =\lim _{T \longrightarrow \infty} \frac{\sum_{k=1}^{K(T)} r_{k}}{\sum_{k=1}^{K(T)} s_{k}} \\
& =\lim _{T \longrightarrow \infty} \frac{\frac{1}{K(T)} \sum_{k=1}^{K(T)} r_{k}}{\frac{1}{K(T)} \sum_{k=1}^{K(T)} s_{k}} \\
& =\frac{E[R]}{E[S]}=\frac{V_{\max }-V_{\min }}{\ln \left(\frac{V_{\max }}{V_{\min }}\right)} .
\end{aligned}
$$

Here $K(T)$ is the total number of trips taken within time $T$, including the last one that may be incomplete. $r_{k}$ and $s_{k}$ are the travel distance and the travel time of the $k^{t h}$ trip, respectively. Note that $\left\{r_{k}\right\}_{k=1,2, \ldots}$ and $\left\{s_{k}\right\}_{k=1,2, \ldots}$ are iid sequences. Assuming that the ensemble average equals time average as $t \rightarrow \infty$, (6) can also be taken as the steady state expected node speed.

From the distribution $f_{S}(s)$, we see that as $V_{\min }$ approaches 0 , the tail distribution of $f_{S}(s)$ decays approximately according to $\frac{c}{s^{2}}$ for some constant $c$ as $s \rightarrow \infty$. Indeed, if $V_{\text {min }}=0$ then 
$T$ has a heavy-tailed distribution [10] since the tail of $f_{S}(s)$ becomes $\frac{2 R_{\max }}{3 V_{\max }} \frac{1}{s^{2}}$. Subsequently, from Equation (5) $E[S] \rightarrow$ $\infty$ as $V_{\min } \rightarrow 0$, and from (6) $\bar{V} \rightarrow 0$ as $V_{\text {min }} \rightarrow 0$. In other words, as the minimum speed approaches zero, the travel time has a higher and higher probability of being very large, with an expected travel time approaching infinity. At the same time, the expected node speed approaches zero. ${ }^{1}$

Comparing the average speed $\bar{V}$ to the initial average speed $\bar{V}_{\text {init }}$ defined as $\frac{V_{\max }+V_{\min }}{2}$ and setting $\alpha=\frac{V_{\max }}{V_{\min }}>1$, we have

$$
\begin{aligned}
\frac{\bar{V}}{\bar{V}_{\text {init }}} & =\frac{\frac{V_{\max }-V_{\min }}{\ln \frac{V_{\max }}{V_{\min }}}}{\frac{V_{\max }+V_{\min }}{2}} \\
& =\frac{2\left(\frac{V_{\max }}{V_{\min }}-1\right)}{\left(\frac{V_{\max }}{V_{\min }}+1\right) \ln \left(\frac{V_{\max }}{V_{\min }}\right)} \\
& =\frac{2(\alpha-1)}{(\alpha+1) \ln \alpha}=g(\alpha)
\end{aligned}
$$

Note that $g^{\prime}(\alpha)<0(\alpha>1), \lim _{\alpha \rightarrow 1} g(\alpha)=1$ and $\lim _{\alpha \rightarrow \infty} g(\alpha)=0$. Therefore

$$
\bar{V} \leq \bar{V}_{\text {init }} \quad \text { (equality holds when } V_{\max }=V_{\min } \text { ). }
$$

This means that the average nodal speed over time is always less than the initial average speed, unless the speed is constant. This also means that there will always be a period of speed decay at the beginning of the simulation until the average speed settles around $\bar{V}$. The distinction is that if $V_{\min }$ takes a positive value, then the average speed eventually stabilizes to a positive $\bar{V}$, whereas if $V_{\min }=0$ then the average speed will continue to decrease as time goes on. Even when $V_{\min }$ is positive, it may take a long time before the average node speed stabilizes if $V_{\min }$ is very small. In general, the smaller $V_{\min }$, the longer the decay period.

The analysis and discussions in this section suggest that in order to reach a positive average speed, one solution is to specify a positive minimum speed for the mobility model. This minimum speed cannot be too close to zero because it is desirable for any simulation model to reach stability as soon as possible. Subsequently, for simulation comparison purposes, the average nodal speed has to be carefully calculated rather than taking the simple average of maximum and minimum speed values. Table I shows a list of speed ranges and the corresponding speed averages. $\bar{V}_{i n i t}$ is the initial average speed defined above. $\bar{V}$ is the steady-state average speed calculated by Eqn.(6) assuming (A1)-(A3). $\bar{V}_{\text {sim }}$ is the average speed of 50 nodes in a 1000 -second simulation after deleting the first 500-second warm-up period. Simulation results are averaged over 10 different scenarios generated by the random waypoint model in a $1500 \mathrm{~m} \times 500 \mathrm{~m}$ area with the modification of positive minimum speed. $\sigma$ is the standard deviation. As shown

\footnotetext{
${ }^{1}$ One can easily check using standard methods that if we take two uniformly distributed nonnegative continuous random variables ( $X_{1}$ and $X_{2}$, respectively) and take a third random variable $\left(X_{3}\right)$ to be the ratio of the first two $\left(X_{3}=\right.$ $\left.X_{1} / X_{2}\right)$, then if the denominator random variable $\left(X_{2}\right)$ is defined over an interval containing the origin, $X_{3}$ would indeed be heavy-tail distributed.
}

in Table I, even though our analysis is for an idealized case based on assumptions (A1)-(A3), we see that the analytical result $\bar{V}$ provides very good prediction for the actual average time in a simulation $\bar{V}_{\text {sim }}$ with the modified random waypoint model. Note that under our assumptions $\bar{V}$ is independent of the range of travel distance $R_{\max }$. This may not be the case for a rectangular area. It is also possible that our approximation is only good for rectangular areas that are not too "narrow". These issues are currently being investigated.

Our results also suggest that simulations need to be "warmed up" properly to eliminate the initial drop in average node speed, i.e., data should not be collected until the average speed has converged around $\bar{V}$ from $\bar{V}_{\text {init }}$. It is a common practice in the scientific simulation community to warm-up a simulation (also known as initial data deletion [11]) aimed at eliminating the effect of the transient part by discarding the data from the initial period of a certain length. This is done to ensure that the system being simulated has entered steady state. In general simulations of mobile systems and ad hoc routing protocols need to be warmed up to get past the initial "cold state" of the system. The discussion here provides another reason why a simulation using such mobility models needs to be warmed-up so that the average nodal speed converges. The actual amount to be discarded in this particular case depends on the minimum speed, which will be discussed more in Section IV.

TABLE I

$$
\bar{V}_{i n i t}, \bar{V} \text {, AND } \bar{V}_{s i m} \text { OF VARIOUS SPEED RANGE }(\text { unit }: \mathrm{m} / \mathrm{s} \text { ) }
$$

\begin{tabular}{|c|c|c|c|}
\hline Speed range & $\bar{V}_{\text {init }}$ & $\bar{V}$ & $\bar{V}_{\text {sim }}$ \\
\hline$[1,19]$ & 10 & 6.11 & $6.17(0.19)$ \\
\hline$[2,18]$ & 10 & 7.28 & $7.33(0.19)$ \\
\hline$[3,17]$ & 10 & 8.07 & $8.00(0.21)$ \\
\hline$[4,16]$ & 10 & 8.66 & $8.70(0.14)$ \\
\hline$[5,15]$ & 10 & 9.10 & $9.09(0.12)$ \\
\hline$[6,14]$ & 10 & 9.44 & $9.45(0.09)$ \\
\hline$[7,13]$ & 10 & 9.69 & $9.69(0.08)$ \\
\hline$[8,12]$ & 10 & 9.87 & $9.87(0.05)$ \\
\hline$[9,11]$ & 10 & 9.97 & $9.97(0.02)$ \\
\hline
\end{tabular}

\begin{tabular}{|c|c|c|c|}
\hline Speed range & $\bar{V}_{\text {init }}$ & $\bar{V}$ & $\bar{V}_{\text {sim }}$ \\
\hline$[1,21]$ & 11 & 6.57 & $6.49(\sigma=0.22)$ \\
\hline$[2,22]$ & 12 & 8.34 & $8.42(0.28)$ \\
\hline$[3,23]$ & 13 & 9.82 & $9.80(0.25)$ \\
\hline$[4,24]$ & 14 & 11.16 & $11.24(0.22)$ \\
\hline$[5,25]$ & 15 & 12.43 & $12.50(0.27)$ \\
\hline$[6,26]$ & 16 & 13.64 & $13.70(0.25)$ \\
\hline$[7,27]$ & 17 & 14.82 & $14.83(0.22)$ \\
\hline$[8,28]$ & 18 & 15.96 & $16.03(0.29)$ \\
\hline$[9,29]$ & 19 & 17.09 & $17.23(0.25)$ \\
\hline$[10,30]$ & 20 & 18.20 & $18.27(0.27)$ \\
\hline
\end{tabular}

\section{IMPROVED MOdELS AND SimUlation}

In this section we propose a simple method, as previously suggested, to modify the random waypoint model so that the mobility scenario reaches a steady state in terms of node speed after a quick warm-up period. We further illustrate the significance of this improvement by comparing simulation results generated by this modified model to that generated by 
the original random waypoint model. We present results for both DSR and AODV over a range of commonly accepted performance metrics. We show that the modified model provides more reliable time-average measures, and that the original model can generate misleading results. However, it is important to note that our simulation is not for the purpose of performance comparison between DSR and AODV, but rather to illustrate how simulation results are affected by the vanishing mobility of the random waypoint model. Both protocols have been continuously updated. We employed DSR code in ns-2 version 2.1b8a [8] and AODV code from [12].

\section{A. Improvement}

There are many potential solutions to the average speed decay problem. Here we examin one of the simplest improvements and leave the discussion on alternative methods to the next section.

Our analysis in section II suggests that one solution is to set a non-zero minimum speed. Our study shows that by doing so the simulation results quickly converge to a constant and stable level. We will define settling time as the time to approach within $10 \%$ of the steady-state average speed. Then the scenario of $[1,19]$ settles after 142 seconds, which is considered to be within a warm-up period during a 900-second simulation. This guarantees the fairness of performance comparison regardless of the simulation time used. Our simulation result is presented in the remainder of this section.

\section{B. Simulation Environment}

To maintain consistency with other research results, we employed the $n s-2$ simulator [8], using the same node movement (each scenario 900 seconds long) and traffic data as in the previous work on performance comparison by Broch et al. [2] as well as our own. In the case of positive minimum speed such as a speed range $[1,19]$, we generated mobility data using a simple modified version of setdest [8]. In these scenarios, 50 nodes move in a $1500 \mathrm{~m} \times 300 \mathrm{~m}$ rectangular area at uniformly distributed speeds. Since pause time does not have a significant effect on our analysis, we set the pause time to zero in all scenarios. For each set of parameters, we ran 30 different scenarios. $^{2}$ Our scenarios consist of various range of speed including $(0,20]$. For traffic data, we also chose the same constant bit rate (CBR) scenario as in [2]. Unlike the previous comparisons by Broch et al. [2] and by Perkins et al. [4] with various number of sources and bit rates, we only used CBR scenarios of 30 sources where each source node transmits data of 64 bytes per packet at a rate of 4 packets per second. This is because the simulation is for illustration only, not for a thorough performance comparison. It is also very important to note that our results are all presented in terms of time rather than pause time which all previous works used. In other words, one curve of our results vs. time corresponds to one point as an average over time in the previous works [2], [4].

\footnotetext{
${ }^{2}$ There are only 10 scenarios of a speed range $(0,20]$ and pause time 0 in [2]. We generated 20 more scenarios and averaged over all 30 scenarios.
}

\section{Metrics}

To compare our work to the results in previous works of performance comparison [2], [4], we adopted the following metrics. We calculate these metrics every 100 seconds - e.g., we get 9 data points for a simulation of 900 seconds - so that we can observe changes in the measures in addition to the single average over the entire simulation duration.

- Average node speed: The average speed of all nodes is calculated every 100 seconds using Eqn.(1). The average node speed is a major factor in our analysis, since how it changes with respect to time affects other metrics.

- The number of routing overhead packets: This includes all packets generated by a routing protocol to discover or maintain routes. Each hop taken by a packet is counted separately. So a packet that traverses four hops counts as four overhead packets. All our simulation results in scenarios with speed range $(0,20]$ and 900 -second runtime agree (as a single total time average) with that by Broch et al. in [2].

- Routing overhead packets in bytes: This metric is the same as the previous one except that it is in bytes rather than number of packets. For example, a 100-byte packet that traverses four hops counts as 400 bytes.

- The number of dropped data packets: Due to an error in the physical layer or upper layers, some of transmitted data packets might not be delivered to a destination node. In this case, a router between a source and a destination discards the packet, and it is counted as one dropped data packet.

- Data packet delay: Packet delay is the time elapsed between transmission of a data packet from a source node and its arrival at the destination node. It is calculated only when a packet is successfully delivered to a destination node. This metric is also computed every 100 seconds and averaged over all packets counted.

\section{Simulation Results}

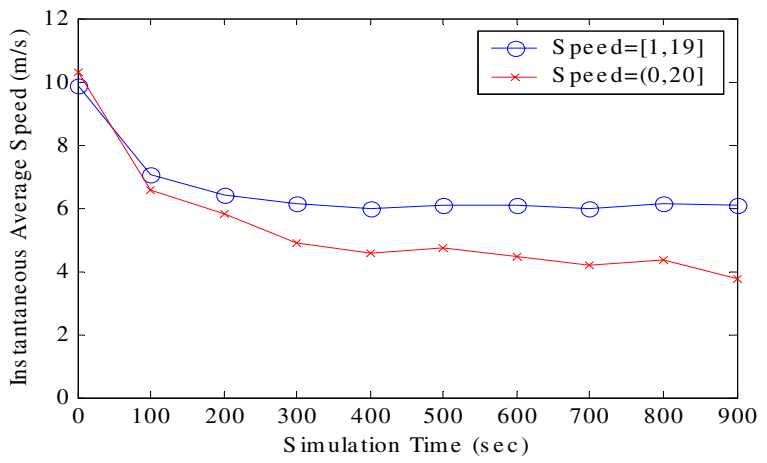

Fig. 4. Average Node Speed in 900-second Simulation

Fig.4 demonstrates how the average node speed changes during the 900 -second simulation as time goes on. As shown in 
Table I, the average node speed in the $[1,19]$ scenario rapidly converges to the expected average speed $6.11 \mathrm{~m} / \mathrm{s}$ and stabilizes at that level, whereas the average node speed in the $(0,20]$ scenario continuously decreases. Since node speed directly affects the performance comparison of ad hoc routing protocols, the decaying average node speed during a simulation will result in varying performance measures over time within the same simulation. If we only look at the time average over total simulation runtime, simulation duration becomes a dominating factor affecting the comparison results.

Our simulation results for DSR and AODV from the 900second scenario with speed range $(0,20]$ and zero pause time, along with results from the 900-second scenario with speed range $[1,19]$ and zero pause time, are shown in Fig.5. We make the following observations.

First, in all four metrics, the performance measures resulting from a speed range $[1,19]$ stabilize after an initial warmup period. On the other hand, all the performance measures resulting from speed range $(0,20]$ continuously decrease as the simulation time progresses. This is particularly clear in routing overhead (Fig.5(a) and (b)) and the number of dropped packets (Fig.5(c)).

Second, in Fig.5(c), after 300 seconds, the difference between DSR and AODV stays relatively constant under speed range $[1,19]$, while speed range $(0,20]$ produces two curves that gradually move closer together and even cross each other at some point due to the decaying speed. On the other hand, the performance of DSR and AODV are nearly indistinguishable under both speed range $[1,19]$ and $(0,20]$ in Fig.5(d).

These observations raise questions on the performance comparison of routing protocols since (1) the performance of routing protocols varies depending on the speed range and (2) the speed range (or the instantaneous speed) drastically and consistently decays even during the same simulation. Our results here imply that the performance measures are closely related to the instantaneous average node speed. Indeed, we believe that how a metric changes with the instantaneous average node speed should be a unique property of a specific ad hoc routing protocol and can be used to fairly compare the performance of different routing protocols. Thus we were motivated to further investigate the relationship between the observed metrics and the instantaneous average node speed.

Fig.6 shows how the metrics of DSR and AODV change as the instantaneous average node speed varies. To obtain various instantaneous average node speeds, different maximum speeds $(1,5,10,15,20,25$, and $30 \mathrm{~m} / \mathrm{s})$ were applied with zero minimum speed. Each maximum speed was used to generate 10 scenarios, so a total of 70 different scenarios were executed. Each point in Fig. 6 corresponds to the average over 10 different scenarios of a specific maximum speed. It can be viewed as plotting Fig.4 (average speed vs. simulation time) and Fig.5 (measures vs. simulation time) together in one plot (measures vs. average speed). As shown in Fig.5, it seems reasonable to discard the first 300 seconds as the warm-up period. Therefore, data from the first 300 seconds were excluded in obtaining the

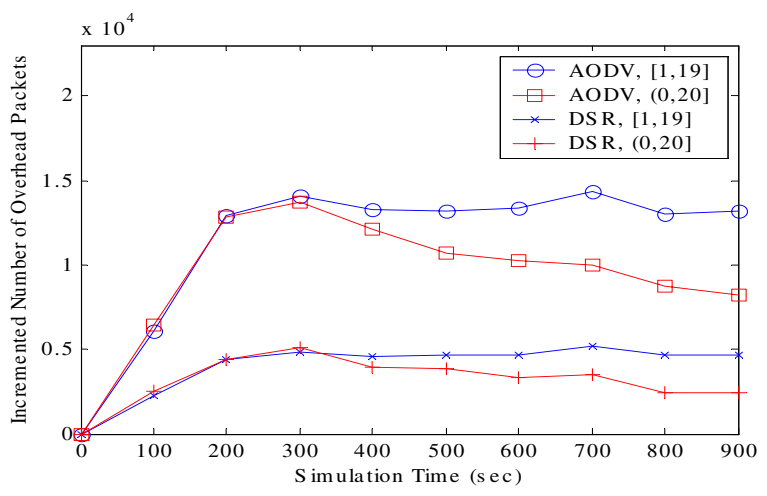

(a) The Number of Routing Overhead Packets

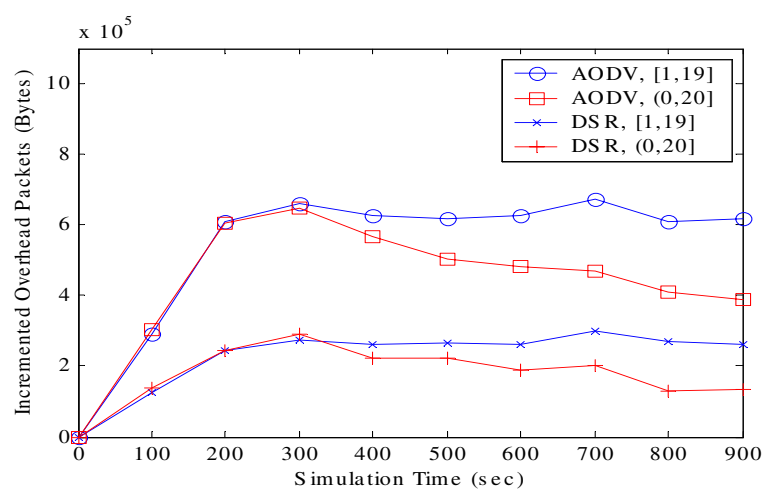

(b) Routing Overhead Packets in Bytes

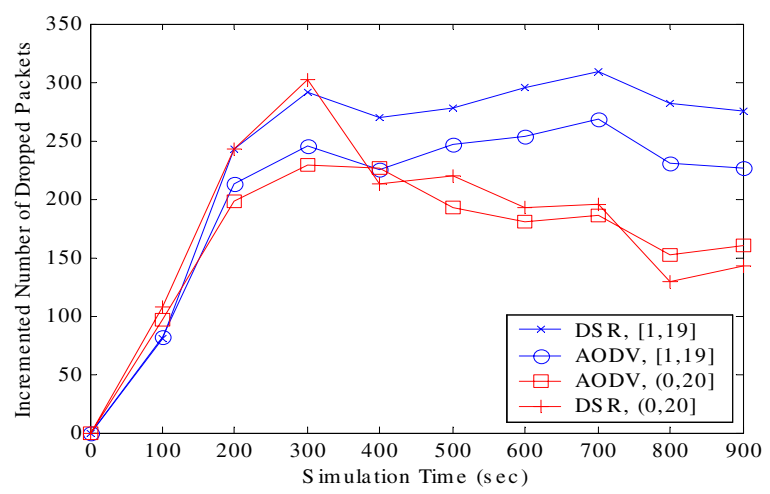

(c) The Number of Dropped Data Packets

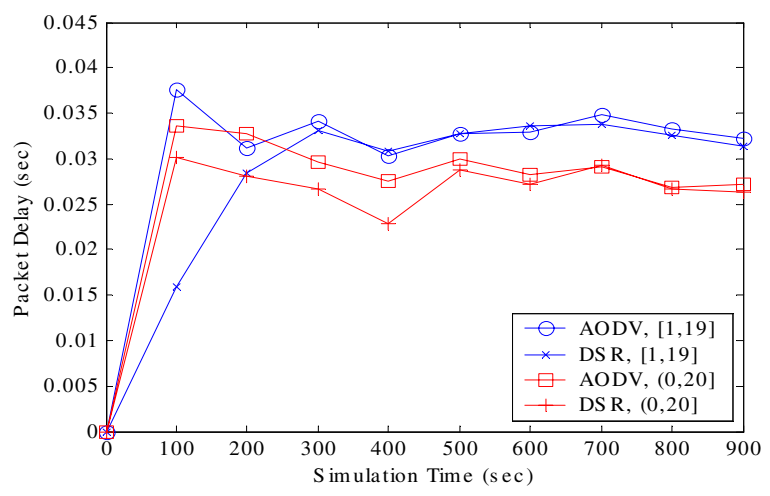

(d) Data Packet Delay

Fig. 5. Incremented Metrics for Every 100 Seconds in 900-second Simulation 


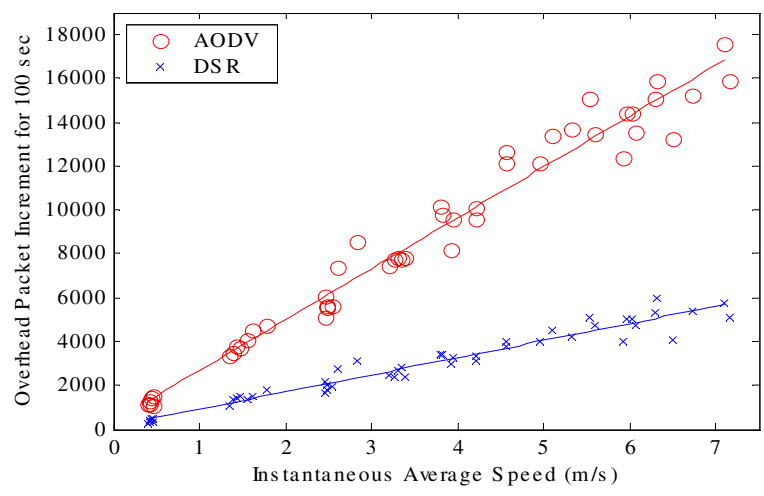

(a) The Number of Routing Overhead Packets

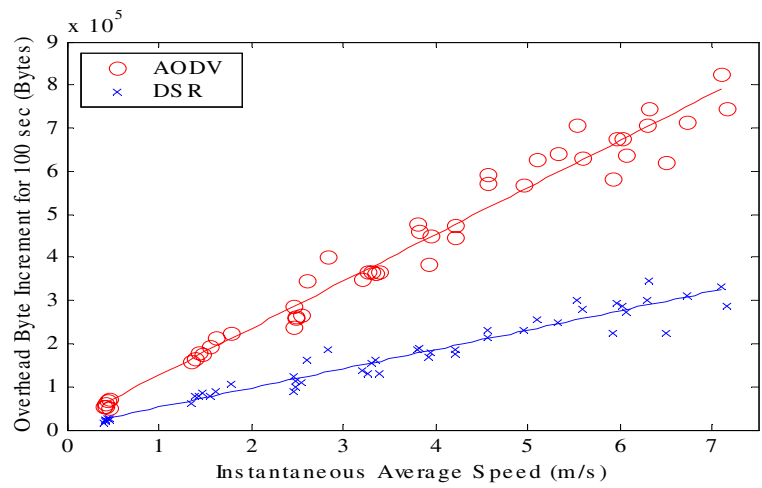

(b) Routing Overhead Packets in Bytes

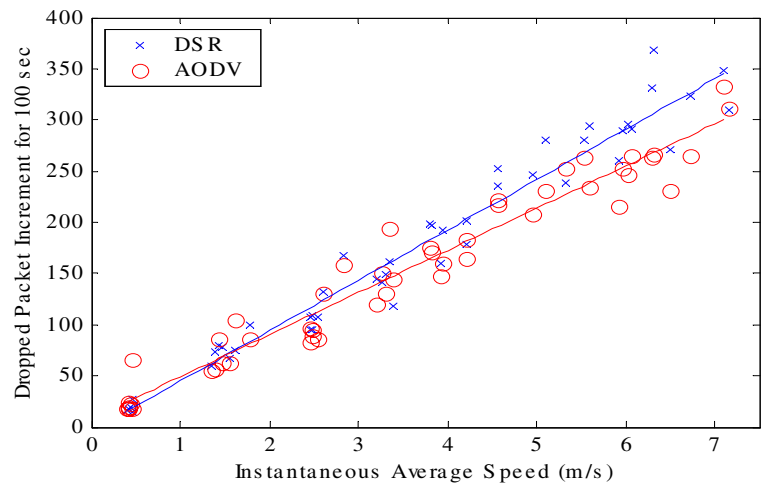

(c) The Number of Dropped Data Packets

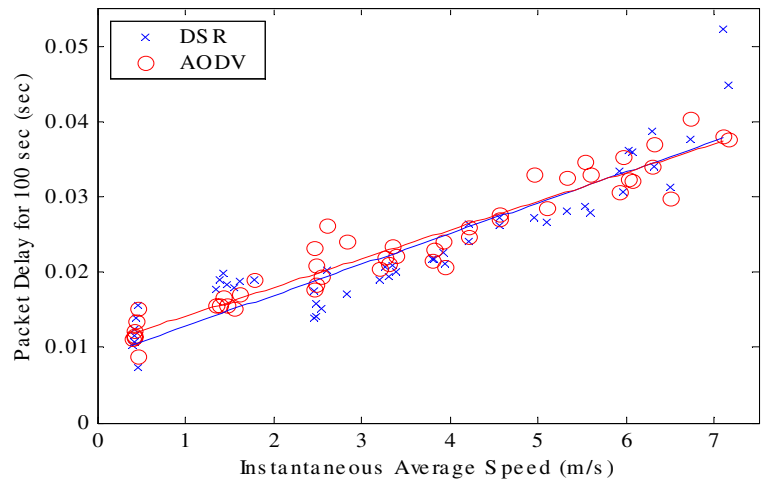

(d) Data Packet Delay

Fig. 6. Performance Metric vs. Instantaneous Average Node Speed results shown in Fig.6. All lines in Fig.6 were fit to minimize the sum of squared error.

As shown in Fig.6(a) and (b), the routing overhead of DSR and AODV increases linearly as the instantaneous average speed increases. This implies that the ratio of routing overhead between DSR and AODV stays roughly constant even though the actual values of the metric decrease as average speed decreases.

Fig.6(c) accounts for the results in Fig.5(c). As shown in Fig.4, after 300 seconds, the instantaneous average node speed of scenarios $[1,19]$ remains constant at $6.1 \mathrm{~m} / \mathrm{s}$. In Fig.6(c), the difference between DSR and AODV at a speed of $6.1 \mathrm{~m} / \mathrm{s}$ is about 40. Therefore the gap between DSR and AODV with speed range $[1,19]$ in Fig.5(c) is maintained at 40.

At the same time, Fig.6(c) also shows that the difference between DSR and AODV becomes smaller as the speed decreases. Below around $2 \mathrm{~m} / \mathrm{s}$, the difference is almost negligible, indicating that DSR and AODV show nearly the same performance in terms of the dropped data packet (or equivalently, packet delivery ratio) at low speeds. This is why the graphs of speed range $[0,20]$ in Fig.5(c) seem to converge as time progresses.

In Fig.6(d), there is little difference between DSR and AODV over almost all speed range. This implies that the performance of DSR and AODV in terms of the packet delay is roughly the same. However, note that the data points of DSR seem more deviated from the fitted line than that of AODV. We thus applied a higher order curve fitting for the data. A nonlinear fit for data in Fig.6(a)-(c) showed almost no difference from the linear fit, whereas a nonlinear fit for data in Fig.6(d) was slightly different.

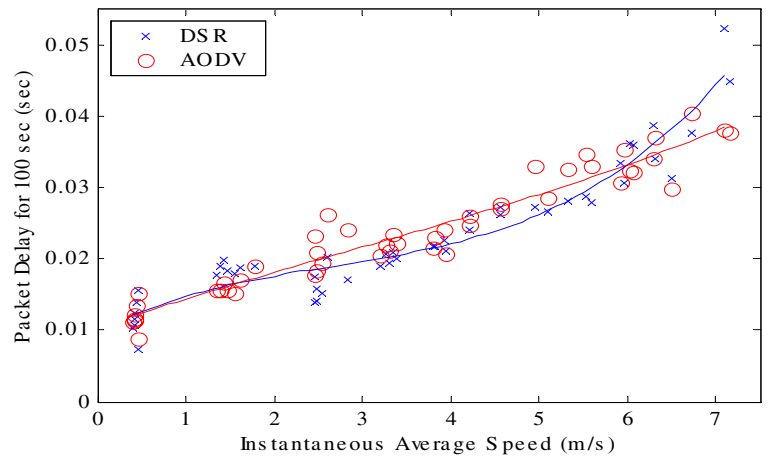

Fig. 7. 3rd Order Curve Fit in Fig.6(d)

Fig.7 is a 3rd order curve fit for the same data shown in Fig.6(d). A distinct feature is that the two performance curves cross twice - at roughly $6.1 \mathrm{~m} / \mathrm{s}$ and $1.5 \mathrm{~m} / \mathrm{s}$, respectively - within the range of instantaneous average node speed we examined. DSR shows a higher packet delay at relatively higher speeds, AODV higher at the intermediate speeds, and the two almost the same at low speeds. Note the gap in packet delay of DSR and AODV shown in Fig.5(d) with speed range $[1,19]$ is almost negligible since there is no difference between them at a speed $6.1 \mathrm{~m} / \mathrm{s}$ in Fig.7. 


\section{E. Summary}

We summarize key conclusions of this section as follows. We have shown that the original random waypoint model with zero minimum speed cannot reach a steady state over the course of a simulation, and the level of mobility continuously decreases. This causes the metrics observed to continuously decrease as well. Because of this, time average of these metrics cannot be reliably compared by varying the maximum speed. As we have seen, even under the same maximum speed depending on how long the simulation is run, the resulting average can drastically differ. Thus under the original random waypoint model, the maximum speed is not a meaningful measure of the level of mobility in the network. For the same reason, the initial average speed is also not a meaningful measure. Note that in this case the steady state average does not exist. On the other hand, by adopting the simple fix proposed in this section which sets a positive minimum speed, the average node speed stabilizes and so do the performance metrics. Time averages can then be computed by discarding the initial period and be reliably compared. Under this modified model, the steady state average node speed is a meaningful measure of the level of mobility that should be used.

\section{Discussion}

\section{A. Alternative Improvements}

There could be many potential solutions to avoid the speed decay as time progresses. In the last section we suggested one of the simplest methods by setting a positive minimum speed. Some other improvements can be derived by considering the reason behind speed decay. Slow-moving nodes maintain low speed for relatively long periods of times until they reach the destination, which cause the decay of average speed. In light of this, we can adopt a strategy analogous to the real life: increase the speed if a destination is far away, and reduce the speed when the destination is near. For example, a speed $v$ can be chosen from a distribution whose mean is correlated with the chosen travel distance. Alternatively, if a steady-state distribution of the average speed can be obtained, we will be able to start the simulation immediately from the steady state. We are currently investigating these alternatives.

\section{B. Decay Time}

The instantaneous average speed $\bar{v}(t)$ defined in Section II is essentially a random process. While it is technically possible to fully characterize this random process and therefore determine precisely how long it takes for the average to converge within a certain percentage of the steady state value, the actual computation turns out to be non-trivial. For the purpose of simulation, however, one could always obtain an empirical estimate on this decay time by pre-running the mobility model. In actual simulations, the amount to discard will also be determined by how long it takes the system being simulated to settle into an equilibrium.

\section{Related Work}

Ad hoc network routing protocols have been well-studied in the past few years. The random waypoint model is often used to evaluate the performance of each particular routing protocol.

Broch et al. [2] used the random waypoint model to compare the performance of DSDV, TORA, DSR, and AODV. They chose packet delivery ratio, routing overhead and path optimality as metrics to compare. However, all metrics were reported as time averages over 900 seconds, while the pause time is varied from experiment to experiment. As we have shown, instantaneous values of these metrics change - some dramatically - over the course of such a simulation.

Perkins et al. [4] performed a similar comparison of DSR and AODV using the random waypoint model. They compared the performance observing different metrics such as average packet delay and normalized routing load. However, results were again represented as averages over time.

A recent paper by Perkins, Hughes and Owen [5] shows that node speed, pause time, network size and the number of sources can affect the performance of routing protocols. Node speed is shown to be a significant factor, while pause time is not. They used random waypont model but employed Global Mobile System Simulator (GloMoSim) rather than $n s-2$. Again, only time averages were reported. In addition, they only ran simulations for 200 seconds, which may not be enough to get past the warm-up period needed.

The random waypoint model itself has also been quite extensively studied. Bettstetter [9] showed by simulation that the random waypoint model does not have a uniform distribution of nodes. Chu and Nikolaidis [6] mathematically showed and confirmed by simulation the same observation. In addition, they showed that there is a relationship between the node distribution and node speed. It was argued that this is partly due to the boundary effect. It was further shown that with different node speed, the mobility scenario poses different connectivity properties. However, such results were again obtained as a time average rather than the change over time. The influence of how nodal speed decay influences connectivity properties remains important future work.

Royer et al. [13] proposed a random direction model to address the non-uniform node distribution problem in the random waypoint model. However, this model suffers from the same vanishing average speed problem since the reason behind speed decay also applies to the random direction model. We indeed observed in simulation that the average node speed under this model decayed in much the same way as in the random waypoint model.

In other recent work, Camp, Boleng, and Davies [7] studied and analyzed a variety of mobility models that have been proposed to date, including the random waypoint model. In particular, they showed the change in average percentage (or number) of neighbors as time progresses. Fig.4 in [7] shows that the average number of neighbors has a stable mean but an increased variance over larger time scales. This is suggestive of decreased movement of nodes (i.e., nodes are more likely to 
remain neighbors or remain neighbors for longer periods due to slow movement), but this was not explicitly pointed out. Various mobility models were also compared in their paper in terms of average speed which is assumed to be the average of minimum and maximum speed rather than the instantaneous node speed.

\section{CONCLUSION}

The random waypoint model is widely used as a mobility model to compare the performance of various mobile ad hoc network routing protocols. In this paper we have shown that the random waypoint model in its current form fails to reach a steady state in terms of instantaneous average node speed, but rather the speed continuously decreases as simulation progresses. Consequently, this model cannot be used to conduct performance evaluation measured as time averages. Such averages are based on metrics that change over time, sometimes substantially. Considering only these averages can result in misleading or incorrect conclusions.

We showed by an intuitive explanation and a formal study the reason behind the speed decay. Based on our analysis we also proposed a simple solution to the problem, which is to set a positive minimum speed. Our improved model is able to quickly converge to a constant speed. Performance measures resulting from this improvement were also able to stabilize. Our analysis, although based on simplifying assumptions, can be used to accurately estimate the expected instantaneous average node speed given the minimum and maximum speeds and the area of the mobility scenario.

Conceptually, the problem revealed in this paper is not a consequence of the random waypoint model itself. In other words, the rationale for the random waypoint model does not limit us from choosing a minimum speed other than zero. For that reason the simple improvement we presented in this paper may still be considered a "random waypoint model". It is a common belief that one can set the minimum speed to zero. It is this belief and the subsequent wide application of it, which made zero the default minimum speed, that have led to this problem and highlight the significance of our work.

\section{APPENDIX}

\section{A. Distribution of travel distance $R$}

The probability density of any location $(x, y)$ within a circle of radius $R_{\max }$ is $\frac{1}{\pi R_{\max }^{2}}$. The travel distance $R$ therefore has the following probability distribution:

$$
\begin{aligned}
P(R \leq r) & =\int_{0}^{2 \pi} \int_{0}^{r} \frac{1}{\pi R_{\max }^{2}} r^{\prime} d r^{\prime} d \theta \\
& =\frac{r^{2}}{R_{\max }^{2}}
\end{aligned}
$$

The pdf of $R$ is thus

$$
f_{R}(r)=\frac{\partial P}{\partial r}=\frac{2 r}{R_{\max }^{2}}, 0 \leq r \leq R_{\max } .
$$

The expectation of $\mathrm{R}$ is then

$$
E[R]=\int_{0}^{R_{\max }} r \frac{2 r}{R_{\max }^{2}} d r=\frac{2}{3} R_{\max }
$$

\section{B. Distribution of travel time $S$}

Under assumption (A3), speed $V$ is uniformly distributed from $V_{\min }$ to $V_{\max }$. Thus $f_{V}(v)=\frac{1}{V_{\max }-V_{\min }}$.

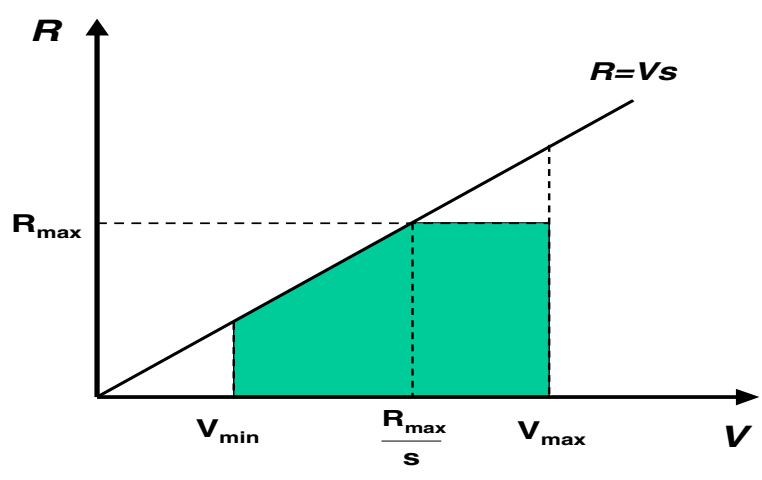

Fig. 8. Distance-Speed Graph

We compute the probability distribution of $S, P(S \leq s)$ below by following the illustration in Fig. 8.

(i) $R_{\max } \leq V_{\min } s \quad\left(s \geq \frac{R_{\max }}{V_{\min }}\right)$

$$
\begin{aligned}
P(S \leq s) & =P\left(\frac{R}{V} \leq s\right)=P(R \leq V s) \\
& =\int_{V_{\min }}^{V_{\max }} \int_{0}^{R_{\max }} f_{R, V}(r, v) d r d v=1 .
\end{aligned}
$$

(ii) $V_{\min } s \leq R_{\max } \leq V_{\max } s \quad\left(\frac{R_{\max }}{V_{\max }} \leq s \leq \frac{R_{\max }}{V_{\min }}\right)$

$$
\begin{aligned}
P & S \leq s)=P(R \leq V s) \\
= & \int_{V_{\min }}^{\frac{R_{\max }}{s}} \int_{0}^{v s} f_{R, V}(r, v) d r d v \\
& +\int_{\frac{R_{\max }}{s}}^{V_{\max }} \int_{0}^{R_{\max }} f_{R, V}(r, v) d r d v \\
= & \int_{V_{\min }}^{\frac{R_{\max }}{s}} \int_{0}^{v s} \frac{2 r}{R_{\max }^{2}\left(V_{\max }-V_{\min }\right)} d r d v \\
& +\int_{\frac{R_{\max }}{V_{\max }}}^{R_{\max }}\left\{\frac{2 r}{R_{\max }^{2}} \frac{1}{\left(V_{\max }-V_{\min }\right)}\right\} d r d v \\
= & \frac{2 R_{\max }}{3\left(V_{\max }-V_{\min }\right)} \frac{1}{s}-\frac{V_{\min }^{3}}{3 R_{\max }^{2}\left(V_{\max }-V_{\min }\right)} s^{2} \\
& +\frac{V_{\max }}{V_{\max }-V_{\min }} .
\end{aligned}
$$




$$
\begin{aligned}
\text { (iii) } R_{\max } \geq & V_{\max } s \geq 0 \quad\left(0 \leq s \leq \frac{R_{\max }}{V_{\max }}\right) \\
P(S \leq s) & =P(R \leq V s) \\
& =\int_{V_{\min }}^{V_{\max }} \int_{0}^{v s} f_{R, V}(r, v) d r d v \\
& =\int_{V_{\min }}^{V_{\max }} \int_{0}^{v s} \frac{2 r}{R_{\max }^{2}\left(V_{\max }-V_{\min }\right)} d r d v \\
& =\frac{s^{2}\left(V_{\max }^{2}+V_{\min }^{2}+V_{\max } V_{\min }\right)}{3 R_{\max }^{2}} .
\end{aligned}
$$

By differentiating $P(S \leq s)$ with respect to $s$, we obtained the pdf of travel time $S$ as follows. (Eqn.(4) in Section II.B)

$$
f_{S}(s)=\left\{\begin{array}{cl}
\frac{2 s}{3 R_{\max }^{2}}\left(V_{\max }^{2}+V_{\min }^{2}\right. & 0 \leq s \leq \frac{R_{\max }}{V_{\max }} \\
\frac{\left.+V_{\max } V_{\min }\right)}{2 R_{\max }} \frac{1}{3\left(V_{\max }-V_{\min }\right)}-v^{2} & \frac{R_{\max }}{V_{\max }} \leq s \leq \frac{R_{\max }}{V_{\min }} \\
\frac{2 V_{\min }^{3}}{3 R_{\max }^{2}\left(V_{\max }-V_{\min }\right)} s & s \geq \frac{R_{\max }}{V_{\min }}
\end{array}\right.
$$

\section{The expectation of travel time $S$}

The expectation of travel time $S$ is then computed from this pdf as follows. (Eqn.(5) in Section II.B)

$$
\begin{aligned}
E[S]= & \int_{0}^{\infty} s \cdot f_{S}(s) d s \\
= & \int_{0}^{\frac{R_{\max }}{V_{\max }}} s \cdot \frac{2\left(V_{\max }^{2}+V_{\min }^{2}+V_{\max } V_{\min }\right)}{3 R_{\max }^{2}} s d s \\
& +\int_{\frac{R_{\max }}{V_{\max }}}^{\frac{R_{\max }}{V_{\min }}} s \cdot\left\{\frac{2 R_{\max }}{3\left(V_{\max }-V_{\min }\right)} \frac{1}{s^{2}}\right. \\
& \left.-\frac{2 V_{\min }^{3}}{3 R_{\max }^{2}\left(V_{\max }-V_{\min }\right)} s\right\} d s \\
= & \frac{2 R_{\max }}{3\left(V_{\max }-V_{\min }\right)} \ln \left(\frac{V_{\max }}{V_{\min }}\right) .
\end{aligned}
$$

\section{REFERENCES}

[1] D. B. Johnson and D. A. Maltz, "Dynamic source routing in ad hoc wireless networks," in Mobile Computing, Imielinski and Korth, Eds. Kluwer Academic Publishers, 1996, vol. 353.

[2] J. Broch, D. A. Maltz, D. B. Johnson, Y.-C. Hu, and J. Jetcheva, "A performance comparison of multi-hop wireless ad hoc network routing protocols," in Mobile Computing and Networking (MobiCom), 1998, pp. $85-97$.

[3] C. E. Perkins and E. M. Royer, "Ad-hoc on-demand distance vector routing," in Proceedings of the 2nd IEEE Workshop on Mobile Computing Systems and Applications, New Orleans, LA, February 1999, pp. 90-100.

[4] C. E. Perkins, E. M. Royer, S. R. Das, and M. K. Marina, "Performance comparison of two on-demand routing protocols for ad hoc networks," IEEE Personal Communications, vol. 8, no. 1, pp. 16-28, February 2001.

[5] D. D. Perkins, H. D. Hughes, and C. B. Owen, "Factors affecting the performance of ad hoc networks," in Proceedings of the IEEE International Conference on Communications (ICC), 2002.

[6] T. Chu and I. Nikolaidis, "On the artifacts of random waypoint simulations," in Proceedings of the 1st International Workshop on Wired/Wireless Internet Communications (WWIC2002), in conjunctions with the International Conference on Internet Computing (IC'02)., 2002.
[7] T. Camp, J. Boleng, and V. Davies, "Mobility models for ad hoc network simulations," in Wireless Communication and Mobile Computing (WCMC): Special issue on Mobile Ad Hoc Networking: Research, Trends and Applications, 2002.

[8] "The network simulator - ns-2," http://www.isi.edu/nsnam/ns/, 2002.

[9] C. Bettstter, "Smooth is better than sharp: a random mobility model for simulation of wireless network," in Proceedings of the ACM International Workshop on Modeling, Analysis and Simulation of Wireless and Mobile Systems, 2001.

[10] W. Stallings, High-Speed Networks, TCP/IP and ATM design principles. Prentice Hall, 1998.

[11] R. Y. Rubinstein, Simulation and the Monte Carlo method. John Wiley \& Sons, 1981.

[12] "Aodv - simulation code," http://moment.cs.ucsb.edu/AODV/aodv.html, 2002.

[13] E. M. Royer, P. M. Melliar-Smith, and L. E. Moser, "An analysis of the optimum node density for ad hoc mobile networks," in Proceedings of the IEEE International Conference on Communications (ICC), Helsinki, Finland, June 2001. 